\title{
Clear Cell Sarcoma of Soft Tissue: A Rare Malignancy Entity
}

\author{
Kenza Kandri Rody ${ }^{1}$, Soumia Faras ${ }^{1}$, Najat Elaattoul ${ }^{1}$, Imane Boujguenna ${ }^{2}$, Hanane Rais ${ }^{2}$, Ouafa Hocar ${ }^{1}$, Said Amal ${ }^{1}$
}

${ }^{1}$ Dermatology-Venerology department; Mohammed VI university hospital center; Marrakesh Morocco

${ }^{2}$ Pathological anatomy department; Mohammed VI university hospital center; Marrakesh Morocco

DOI: $10.36347 /$ sjmcr.2021.v09i03.027

| Received: 02.02.2021 | Accepted: 08.03.2021 | Published: 28.03.2021

*Corresponding author: Kenza Kandri Rody

Abstract

Clear cell sarcoma (CCS) of soft tissue or soft tissue melanoma is a rare malignant tumor in young adults, preferentially affecting the lower limbs. We report a new observation of a 17-year-old patient, without a significant history, consulted for a nodular lesion of the left ankle, painless, appeared 06 months before, evolving in a context of general impairment. The clinical examination found a purplish nodule, about $2 / 2 \mathrm{~cm}$, hard, fixed, associated with multiple nodular lesions along the homolateral lower limb, with the presence of homolateral inguinal lymphadenopathy. There was no associated motor or vascular disorder. The nodule ultrasound showed a mass of soft tissue, hypoechogenic, heterogeneous. The computed tomography of the ankle revealed a lesional process of the medial face infiltrating the tendon and ligament structures and encompassing the posterior tibial artery. Histological examination showed round cell malignant tumor proliferation. The immuno-histochemical supplement showed an expression of the S100 protein and monoclonal antibody HMB-45 by tumor cells. The biopsy of an inguinal node affirmed its metastatic nature. The anatomo-clinical confrontation allowed to retain the diagnosis of clear cell sarcoma. The extension assessment made of a chest-abdominal-pelvic computed tomography showed metastatic lymph nodes intra and retroperitoneal. The patient was given chemotherapy (ifosfamide-adriamycin). Sarcoma with clear soft tissue cells or melanoma of the soft parts is a rare malignant tumor. It occurs most often in young adults at the lower limbs. It is a firm subcutaneous mass, slowly progressive, well limited, usually deep attached to tendon and aponeurotic structures. The positive diagnosis is based on histology coupled with immunohistochemistry. It can be helped by molecular biology in case of doubt diagnosis with melanoma. Its poor prognosis requires early and adequate management.

Keywords: Clear cell sarcoma of soft tissue, Tumors, soft tissue melanoma.

Copyright $(92021$ The Author(s): This is an open-access article distributed under the terms of the Creative Commons Attribution 4.0 International License (CC BY-NC 4.0) which permits unrestricted use, distribution, and reproduction in any medium for non-commercial use provided the original author and source are credited.

\section{INTRODUCTION}

Clear cell sarcoma (CCS) or soft tissue melanoma is a rare entity. It is a malignant tumor of young adults, preferentially affecting the deep soft tissues of the lower limbs. We report a new metastatic CSC observation.

\section{Case Report}

A 17-year-old patient, with no significant history, consulted for a nodular lesion of the left ankle, painless, appeared 06 months earlier, following a minimal trauma, evolving in a context of altered general condition (Figure 1). The clinical examination found a purplish nodule, about $2 / 2 \mathrm{~cm}$, hard, fixed, associated with multiple nodular lesions (erythematous, hard, fixed and painless) without local inflammatory signs of the antero external of the homolateral lower limb of which the largest measured $4 \times 3 \mathrm{~cm}$, along the face with the presence of homolateral inguinal lymphadenopathy. There was no associated motor or vascular disorder. The radiographs of the ankle and right lower limb were free of abnormalities. The nodule ultrasound showed a mass of soft, hypoechogenic, heterogeneous tissues. The computed tomography of the ankle revealed a lesional process of the medial face infiltrating the tendon and ligament structures and encompassing the posterior tibial artery. Histological examination showed round cell malignant tumor proliferation. The immunohistochemical supplement showed expression of the S100 protein and Ag HMB 45 by tumor cells in favor of either a clear cell sarcoma or a melanoma. The biopsy of an inguinal node affirmed its metastatic nature. The anatomo-clinical confrontation allowed to retain the diagnosis of CCS. The extension assessment made of a chest-abdominal-pelvic computed tomography showed 
metastatic lymph nodes intra-and retroperitoneal. The therapeutic decision taken in multidisciplinary consultation was to put the patient on chemotherapy (ifosfamide-Adriamycin protocol). The evolution was marked by tumor progression. The patient had refused second-line chemotherapy.

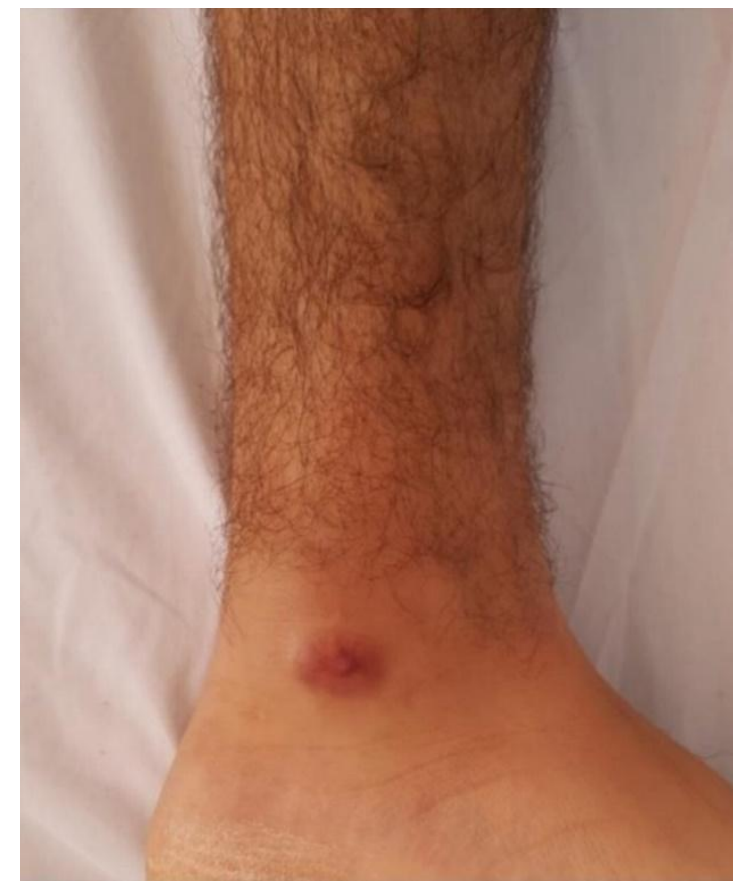

Fig-1: Nodular lesion of the left ankle, hard and fixed

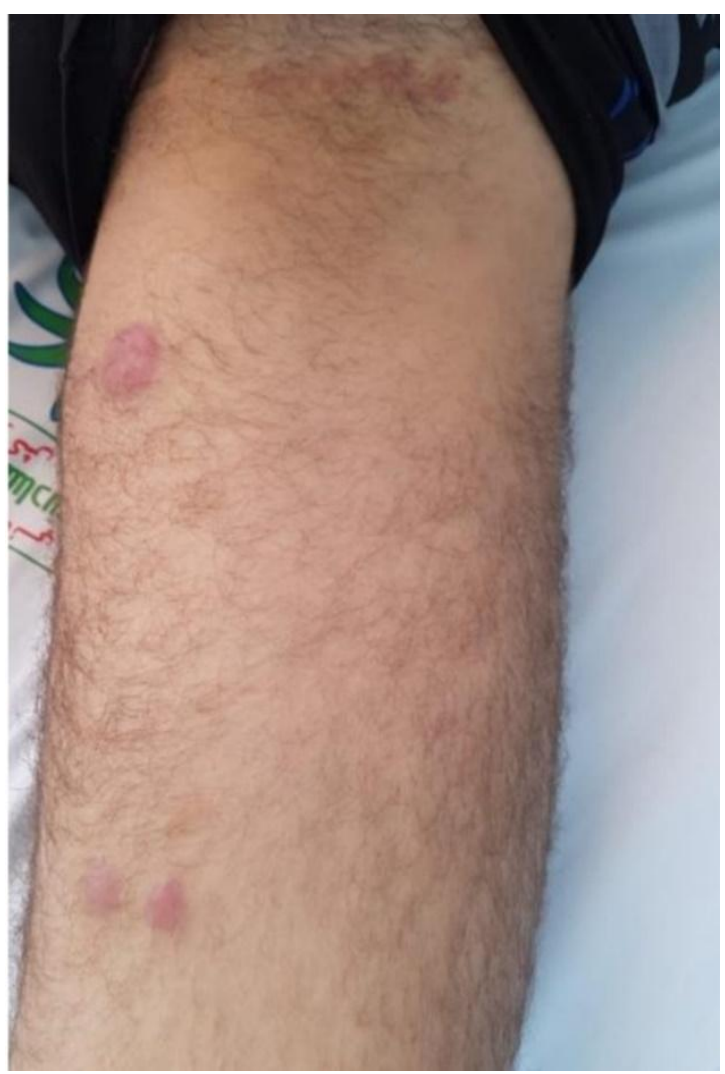

Fig-2: Multiple nodular lesions of the antero external of the homolateral lower limb

\section{DiSCUSSION}

Sarcoma in soft tissue clear cells or soft tissue melanoma is a rare malignant tumor that accounts for only $1 \%$ of all soft tissue sarcomas [1]. It was described for the first time by Enzinger in 1965 and then in 1983, the term soft tissue melanoma was proposed after the finding of melanin in tumor cells [2-10]. Slightly female predominant [2-5], It occurs most often in adolescents and young adults rarely in children under 10 years old or the adult over 50 years old [7-10]. It is primarily located at the extremities adjacent to the ankle and foot (40\% of cases) [2-10]. A history of trauma may be present $[5,8]$ as in our case.

The typical clinical presentation is the appearance of a firm subcutaneous mass, well limited, partially encapsulated, of whitish grey, lobed or multinodular color and usually deep attached to the tendino-aponevrotic structures $[2,4,5,8]$. Tumor size ranges from 1 to $15 \mathrm{~cm}$ with a median size of 2 to $6 \mathrm{~cm}$ [2-5]. Cystic and necrotic lesions are occasionally observed [2-7]. Pain is reported in 50\% of cases [4-8]. At first slow and then fast growing, metastases are usual and the prognosis remains unfavorable [2-11]. Radiologically, standard radiographs don't show bone abnormalities, but lytic lesions and bone destruction have rarely been described [5]. This is the case with our observation. MRI is of great contribution in early diagnosis by showing a tumor mass in low signal on T1, rising after injection of gadolinium and variable signal in T2 [2, 6]. Histologically, the tumor is made up of nests, bundles or lobules of cells delimited by fibrous septa which are continued by collagen-oriented structures corresponding to tendinous or aponeurotic structures. The cells are monomorphic, polygonal or fusiform. They have a low eosinophilic or clarified, finely granular cytoplasm. The nucleus is round or ovoid, densified at the periphery and centered by a prominent basophilic nucleolus. Mitotic activity is low. Multinucleated giant cells can be seen [2-5, 7-9, 11]. Detection of intracellular melanin by Fontana staining is observed in approximately $50 \%$ of cases $[2-4,7,9$, 11]. Periodic acid schiff stain shows the presence of intra-cytoplasmic glycogen as in our case $[4,7,8]$. The immuno-histochemical study shows in the majority of cases an intense and diffuse cytoplasmic marking of tumor cells by the anti-HMB45 antibody and a less intense and more focal nuclear and cytoplasmic marking by the anti-PS100 antibody. Sarcomatous cells are also positive for vimentin, Melan A, CD99 and specific enolase neuron. Cytokeratin, membrane epithelial antigen, desmin and actin are usually negative [2-5, 7-9]. Cells also have abundant cytoplasm containing numerous mitochondria and glycogen aggregations $[2,4,8]$.

The positive diagnosis is based on histology coupled with immunohistochemistry. Cytogenetic data is a great contribution to make the diagnosis for 
Kenza Kandri Rody et al., Sch J Med Case Rep, Mar, 2021; 9(3): 292-294

difficult cases. Soft Tissue Clear Cell Sarcoma pose a differential diagnosis problem with malignant melanoma due to the similarity of the clinical picture (high rate of lymph node metastases) and histological and immunohistochemical presentation. However, it is distinguished by the absence of pigmented nodules in the skin and the presence in cytogenetic study of a chromosomal translocation (t[12; 22] [q13; q12]) [3,4]. Differential diagnosis can also occur with other types of sarcoma: synovial sarcoma, fibrosarcoma and malignant schwannoma [3, 4, 7, 8]. Its management is complex and multidisciplinary. The treatment is surgical, consisting of a broad excision with possibly lymph node curage, supplemented by radiotherapy and chemotherapy [3, 6-9, 12]. Poor prognosis factors are tumor size greater than $5 \mathrm{~cm}$, necrosis, metastasis and local recurrence [3, 7, 8]. Prognosis is unfavorable (54\% survival) $[3,4]$.

\section{Conclusion}

Clear cell soft tissue sarcoma is a rare melanocytic tumor with a poor prognosis that poses a diagnostic and therapeutic challenge.

\section{REFERENCES}

1. Al Nakshabandi NA, Munk PL. Radiology for the surgeon. Musculoskeletal case 38. J Can Chir. 2007; 50:58-9.

2. Fletcher CDM, Unni KK, Mertens F, editors. World Health Organization classification of tumors. Lyon: Pathology and genetics of tumors of soft tissues and bone IARC Press; 2002

3. Brand`s B, Terrier P, Voigt JJ. Sarcoma has clear soft tissue cells. Ann Pathol. 2000; 20:298-303.

4. Soft Tissue Tumour UPR - Sarcoma Group FNCLCC. 2007; II: 321-3.

5. Mackey SL, Hebel J, Cobb MW. Melanoma of the soft parts (clear cell sarcoma): a case report and review of the literature. J Am Acad Dermatol. 1998; 38:815-9.

6. Kuiper DR, Hoekstrat HJ, Veth RP. The management of clear cell sarcoma. Eur J Surg Oncol. 2003; 29:568-70.

7. Ferlicot S, Guymar S, Chigot JP. Sarcoma has clear cells (soft tissue malignant lanoma) of the calf. Ann Chir. 2001; 126:152-5.

8. Dim DC, Cooley LD, Miranda RN. Clear cell sarcoma of tendons and aponevroses: a review. Arch Pathol Lab Med 2007; 131:152-6.

9. Clark MA, Johnson MB, Thway K. Clear cell sarcoma (melanoma of soft parts): the royal Marsden hospital experience. Eur J Surg Oncol. 2008; 34:800-4.

10. Van Akkooi AC, Verhoef C, Van Geel AN. Sentinel node biopsy for clear cell sarcoma. Eur J Surg Oncol. 2006; 32:996-9.

11. Tyler G, Wirman J, Neale HW. Melanin containing clear cell sarcoma in a fingertip case report and review of the literature. Hand. 1980; 12:308-15.

12. Rivera JC, Lesimple T, Garin E. Malignant melanoma of soft parts. A 'non plastic surgeon's land'? Ann Chir Plast Esthet. 2008; 53:361-40 